\title{
Primordial black holes and remnants of primordial black holes as dark matter: inflationary model building and observational constraints
}

\author{
Ioannis Dalianis* \\ National Technical University of Athens \\ E-mail:dalianis@mail.ntua.gr
}

\begin{abstract}
I discuss the cosmology of Primordial Black Holes (PBHs) produced by density perturbations and consider the scenario that PBHs and the remnants of PBHs constitute a significant fraction of the dark matter in the galaxies. I introduce inflationary models that generate PBHs and, additionally, give rise to fast and slow reheating and kination post-inflationary domination eras. I also present the spectral shapes of gravitational waves (GWs) induced to second order by large scalar perturbations and demonstrate the ability of the GWs to reveal the spectrum of the primordial curvature perturbations at scales much smaller than those directly accessible in the CMB.
\end{abstract}

Corfu Summer Institute 2019 "School and Workshops on Elementary Particle Physics and Gravity" (CORFU2019)

31 August - 25 September 2019

Corfú, Greece

${ }^{*}$ Speaker. 


\section{Introduction}

Nowadays, that the existence of black holes and dark matter are unambiguous and the investigation of the primordial black hole (PBH) dark matter scenario is very motivated. Here, I discuss the PBH and mini-PBH scenario, that realizes the PBH-remnants dark matter scenario. I introduce new and testable inflationary scenarios, that generate PBHs, and assume non-standard cosmological evolution for the early and late universe. I show that the PBHs can be regarded as a powerful tool to probe the primordial fluctuations and give us insights into the dynamics that generated the seeds of the cosmic structure, even if PBHs do not comprise the observed dark matter in the universe. In this context, I also discuss the shape of the induced GWs produced in the early universe and stress that the detection of the induced GW spectrum can be additionally used as a portal to the density perturbations at small scales far beyond the scales directly accessible in the CMB.

This paper is organized as follows. Firstly I present the constraints on the power spectrum of the primordial curvature perturbations coming from the bounds on the PBHs. Next I introduce inflationary models that generate a peak in the scalar power spectrum in accordance with the observational constraints. In the following two sections I discuss the PBH and the PBH-remnants dark matter scenario. Finally, I present the induced gravitational wave (IGWs) spectra predicted by models with an enhanced scalar power spectrum.

\section{Constraints on the primordial curvature perturbations No1: PBHs}

PBHs form from large-amplitude inhomogeneities that decouple from the background expansion and collapse [1]. Roughly, it has to be $G M / R \sim 1$, for a region of mass $M$ over a scale $R$. This can be achieved if the power spectrum $\mathscr{P}_{\mathscr{R}}(k)$ at the inverse scale $R^{-1} \sim k$, characteristic of the PBH mass, has a very large amplitude, much larger than the density perturbation $\delta \rho / \rho \sim 10^{-5}$ measured at the CMB scales. The minimum value for the $\mathscr{P}_{\mathscr{R}}$ required for the realization of a collapse depends on the background pressure as well as the sphericity and the angular momentum of the overdense region. If the $\mathscr{P}_{\mathscr{R}}$ is enhanced at small scales $k^{-1}$ light PBH form; if $k^{-1}$ is large the PBH formed are heavier. This is a key difference compared to stellar black holes that are predicted to be heavier than about few solar masses, $M_{\odot}$.

Steven Hawking predicted that black holes radiate thermally, with a temperature inversely proportional to the mass, $T_{\mathrm{BH}}=\hbar c^{3} /\left(8 \pi G M k_{B}\right)$, and evaporate on a time scale $t_{\mathrm{evap}} \sim G^{2} M^{3} /\left(\hbar c^{4}\right)$, where $M$ the mass of the PBH [2,3]. PBHs with mass $M \lesssim 10^{15} \mathrm{~g}$ evaporate at timescales less than the age of the universe, whereas PBHs with $M>10^{15} \mathrm{~g}$ would still survive today and would be dynamically cold component of the dark matter in galactic structures. Mini PBHs in the range $M \sim 10^{9}-10^{12} \mathrm{~g}$ evaporate during or after the BBN cosmic epoch, and PBHs with mass $M \sim$ $10^{13}-10^{14} \mathrm{~g}$ evaporate during the cosmic epoch of recombination. BBN observables constrain the $\mathrm{PBH}$ abundance [4-11], and the anisotropy damping on CMB observables put the stringent constraint [12]. The abundance of light $\mathrm{PBH}, M>10^{15} \mathrm{~g}$, is constrained from the extra galactic gamma-ray background [13-17].

The PBH dark matter scenario is constrained in a wide range for the mass parameter $M$ by several observational experiments. Black holes of mass above $10^{17} \mathrm{~g}$ are subject to gravitational lensing constraints [18-20]. The CMB constrains the PBH with mass above $10^{33} \mathrm{~g}$ [21]. It has 
been claimed that the CMB bounds on massive PBHs might be relaxed [22-24]. At the large mass region there are also constraints from accretion limits in X-ray and radio observations [25]. For a recent update on the PBH constraints see Ref. [26].

The present relic energy density parameter of primordial black holes with mass $M$ produced at the cosmic time $t$ is

$$
\Omega_{\mathrm{PBH}}(M)=\Omega_{\mathrm{m}} \gamma \beta(M)\left(\frac{M_{\mathrm{rh}}}{M / \gamma}\right)^{\frac{2 w}{1+w}}\left(\frac{M_{\mathrm{eq}}}{M_{\mathrm{rh}}}\right)^{1 / 2} \tilde{g}\left(g_{*}\right)
$$

where $\tilde{g}\left(g_{*}\right)=2^{1 / 4}\left(g_{*}(t) / g_{*}\left(t_{\mathrm{rh}}\right)\right)^{-p / 4}\left(g_{*}\left(t_{\mathrm{rh}}\right) / g_{*}\left(t_{\mathrm{eq}}\right)\right)^{-1 / 4}$, and $g_{*}$ the thermalized degrees of freedom. The parameter $p$ is equal to 1 for $t>t_{\mathrm{rh}}$ and 0 for $t<t_{\mathrm{rh}} . \Omega_{\mathrm{m}}$ is the total matter density parameter today, $M_{\mathrm{eq}}$ is the horizon mass at the moment of matter radiation equality and $M_{\mathrm{rh}}$ the horizon mass at the moment of reheating.

The $\beta(M)$ is the mass fraction of the universe with horizon mass $M / \gamma$ that collapsed and formed PBHs; it can be interpreted as the black hole formation probability. Assuming Gaussian statistics, for a spherically symmetric region it is

$$
\beta(M)=\int_{\delta_{c}} d \delta \frac{1}{\sqrt{2 \pi \sigma^{2}(M)}} e^{-\frac{\delta^{2}}{2 \sigma^{2}(M)}},
$$

where $\sigma(M)$ is the variance of the density perturbations and $\delta$ is the density contrast. The PBH abundance has an exponential sensitivity to the variance of the perturbations $\sigma(M)$ and the threshold value $\delta_{c}$, which dependents on the equation of state of the background energy density. We assign values to $\delta_{c}$ following the findings of Ref. [27], where, in the comoving gauge, it is $\delta_{c}=$ $(3(1+w) /(5+3 w)) \sin ^{2} \frac{\pi \sqrt{w}}{1+3 w}$. For $w=1 / 3$ it is $\delta_{c}=0.41$, and decreases significantly for softer equation of states. For $w \rightarrow 0$ the Eq. (2.2) stops being valid. Employing the results of Ref. [28] for spinless gravitational collapse during MD era the formation rate, is given by

$$
\beta_{\mathrm{MD}}(M) \simeq 0.056 \sigma^{5}(M) .
$$

The PBH production rate is suppressed when the collapsing region has spin [29],

$$
\beta_{\mathrm{MD}}(M)=2 \times 10^{-7} f_{q}\left(q_{c}\right) \mathscr{I}^{6} \sigma(M)^{2} e^{-0.147 \frac{\mathscr{G}^{4 / 3}}{\sigma(M)^{2 / 3}}} .
$$

Benchmark values are $q_{c}=\sqrt{2}, f_{q} \sim 1$ and $\mathscr{I}$ is a parameter of order unity [29]. According to [29] this expression applies for $\sigma(M) \lesssim 0.005$, whereas the equation $\beta_{\mathrm{MD}}(M) \simeq 0.056 \sigma^{5}(M)$ applies for $0.005 \lesssim \sigma(M) \lesssim 0.2$.

The observational constraints put upper bounds on the $\beta(M)$. The size of the $\beta$, as discussed above, depends on the variance $\sigma$ of the density perturbations in a different way if the collapse takes place in a background with or without pressure. If there is thermal pressure the collapse is effectively instantaneous whereas, if the there is no pressure the collapse has a finite duration determined by the $\sigma$ value. Also, in MD era the size of the variance determines whether the PBH formation-rate is affected by the spin. Taking all these into account, the maximum value for the $\mathscr{P}_{\mathscr{R}}(k)$ is derived. Schematically it is

$$
\Omega_{\mathrm{PBH}} \propto \sqrt{\mathscr{P}_{\mathscr{R}}} e^{-\delta_{c}^{2} / \mathscr{P}_{\mathscr{R}}}, \quad \Omega_{\mathrm{PBH}} \propto \mathscr{P}_{\mathscr{R}}{ }^{5 / 2} .
$$


The expression on the left hand side is for collapse during radiation and on the right hand side for spinless collapse during matter era. The bounds on the $\mathrm{PBH}$ abundance translate into bounds on the $\mathscr{P}_{\mathscr{R}}(k)$, that are different for different cosmic eras that the collapse takes place. It is actually known that the equation of state of the Universe has not been directly probed for times prior to BBN $t \sim 1$ $\mathrm{s}$ [30] and a general equation of state $w$ has to be assumed. Using the relation $f=k /(2 \pi)$ we find the frequency-PBH mass correspondence for general equation of state $w$ and reheating temperature $T_{\mathrm{rh}}$,

$$
\begin{aligned}
& k\left(M, T_{\mathrm{rh}}, w\right) \simeq 2 \times 10^{17} \mathrm{Mpc}^{-1}\left(\frac{T_{\mathrm{rh}}}{10^{10} \mathrm{GeV}}\right)^{\frac{1-3 w}{3(1+w)}} \\
&\left(\frac{M / \gamma}{10^{12} \mathrm{~g}}\right)^{-\frac{3 w+1}{3(1+w)}}\left(\frac{g_{*}}{106.75}\right)^{\frac{1}{4} \frac{1-3 w}{3(1+w)}} \tilde{R}(g, w)
\end{aligned}
$$

where $\tilde{R}(g, w)=\left(g_{*} / 106.75\right)^{-1 / 12}$ for $w=1 / 3$ and one for $w \neq 1 / 3$. In the above relation we have assumed a one-to-one correspondence between $k$ and the PBH mass $M$. This it true for the approximation of a monochromatic PBH mass spectrum, which is practically the case in many models.

Assuming a radiation dominated phase in the early universe, with $T_{\mathrm{rh}}>2.2 \times 10^{9} \gamma^{1 / 2} \mathrm{GeV}$, the stringent constraint, coming from the damping of the CMB anisotropies, is on the abundance of mini PBHs with mass $M=M_{\mathrm{cmb}}=2.5 \times 10^{13} \mathrm{~g}$ gives us the bound in the variance of the density perturbations,

$$
\sigma\left(1.9 \times 10^{16}\right) \lesssim 0.035\left(\frac{\delta_{c}}{0.41}\right)\left[1+0.023 \ln \left(\frac{\gamma}{0.2}\right)\right]^{-1 / 2}
$$

Assuming kination domination, $w=1$, with reheating temperatures $T_{\mathrm{rh}} \lesssim 2 \times 10^{9} \mathrm{GeV}$ the $\mathrm{CMB}$ constraint, that applies on the scale with wavenumber $k\left(M_{\mathrm{cmb}}, T_{\mathrm{rh}}\right)$, is

$$
\sigma\left(k\left(M_{\mathrm{cmb}}, T_{\mathrm{rh}}\right) \lesssim 0.032\left(\frac{\delta_{c}}{0.375}\right)\right.
$$

According to the Eq. (2.6), it is $k\left(M_{\mathrm{cmb}}, T_{\mathrm{rh}}\right) \simeq 5 \times 10^{18} k_{*} \gamma^{2 / 3}\left(T_{\mathrm{rh}} / 10^{7} \mathrm{GeV}\right)^{-1 / 3}\left(g_{*} / 106.75\right)^{-1 / 12}$ Assuming a matter domination early universe era, $w=0$, reheated at temperatures $T_{\mathrm{rh}} \lesssim$ $10^{7} \mathrm{GeV}$ and considering spin effects, the variance of the density perturbations has to satisfy

$$
\begin{aligned}
& \sigma\left(k\left(M_{\mathrm{cmb}}, T_{\mathrm{rh}}\right)\right) \lesssim \operatorname{Exp}\left[-6.9-0.09 \ln \frac{T_{\mathrm{rh}}}{\mathrm{GeV}}\right. \\
& \left.+2 \times 10^{-3}\left(\ln \frac{T_{\mathrm{rh}}}{\mathrm{GeV}}\right)^{2}-3 \times 10^{-5}\left(\ln \frac{T_{\mathrm{rh}}}{\mathrm{GeV}}\right)^{3}\right]
\end{aligned}
$$

where $k\left(M_{\mathrm{cmb}}, T_{\mathrm{rh}}\right) \simeq 3 \times 10^{17} k_{*} \gamma^{1 / 3}\left(T_{\mathrm{rh}} / 10^{7} \mathrm{GeV}\right)^{1 / 3}\left(g_{*} / 106.75\right)^{1 / 12}$.

The constraints (2.7), (2.8) and (2.9) can be translated into constraints on the primordial power spectrum $\mathscr{P}_{\mathscr{R}}(k)$ as explained in detail in Ref. [31]. 

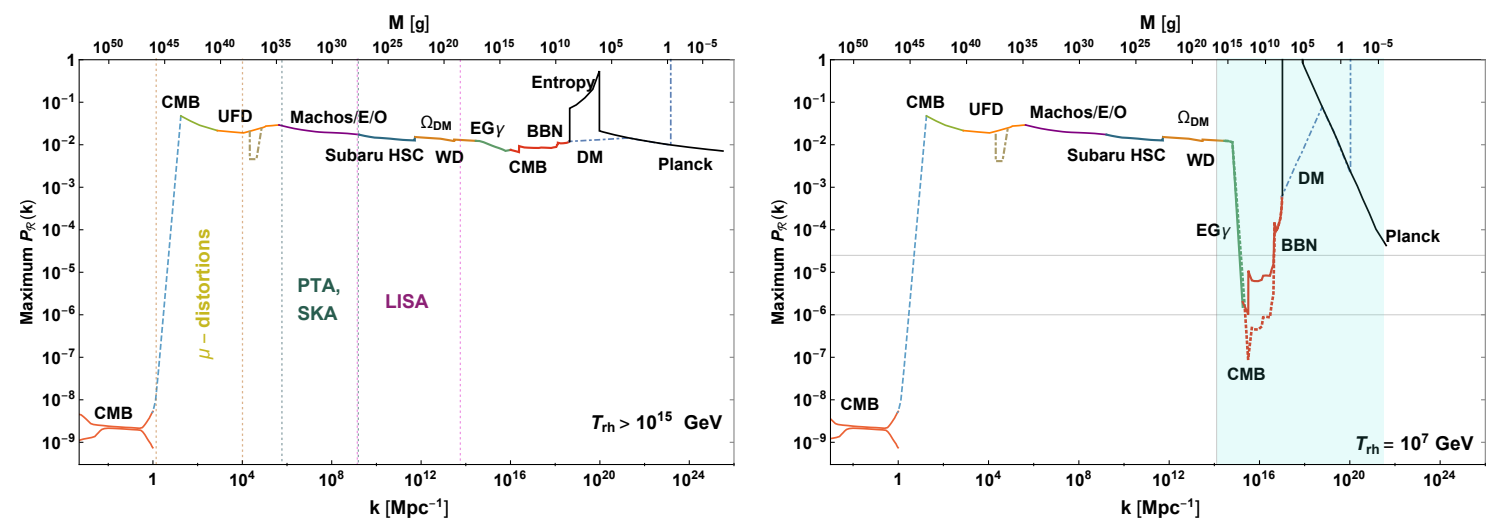

Figure 1: The plots depict upper bounds on the power spectrum of the comoving curvature perturbation coming from constraints on evaporated and nonevaporated PBHs for different early universe cosmic histories after the assumption that $\mathscr{P}_{\mathscr{R}}(k)=\theta^{-2} \sigma_{\max }^{2}(k)$, where $\theta=2 / 5$ and $4 / 9$ for radiation domination era with $T_{\mathrm{rh}} \gtrsim 10^{15} \mathrm{GeV}$, (left panel), and matter domination era with $T_{\mathrm{rh}}=10^{7} \mathrm{GeV}$, (right panel). The blue shaded areas correspond to a scalar-condensate dominated non-thermal phase, caused either by the inflaton or a modulus field. The dashed lines give the upper bounds for spinless gravitational collapse. The CMB bound (red line) is the stringent bound on the small scales.

\section{Inflationary model building}

In order for a model of inflation to seed the PBH formation, a mechanism is needed for the enhancement of the power spectrum of the curvature perturbation at small scales. The power spectrum has to be normalized at the CMB scales by the amplitude of the temperature anisotropies, $\mathscr{P}_{\mathscr{R}} \sim 10^{-9}$, and increased several orders of magnitude at much smaller scales, relevant to the PBH formation. This striking change of the curvature power spectrum with respect to the scale inevitably has to be attributed to some peculiar feature of the inflationary dynamics. The power spectrum, according to the standard approximate analytic estimation, has a dependence $\mathscr{P}_{\mathscr{R}} \sim H^{2} / \varepsilon_{1}$, where $\varepsilon_{1}$ is the first Hubble flow function and $H$ the Hubble parameter. An enhancement in $\mathscr{P}_{\mathscr{R}}$ may take place if the $\varepsilon_{1}$ decreases substantially during the inflationary evolution. In the single field inflationary set up, this can be achieved if there is a near-inflection point about a local plateau for the inflationary potential [32,33]. Another suggestion is an enhanced friction [34] for the inflaton field $[35,36]$. Hence, let us discuss $\mathscr{P}_{\mathscr{R}}(k)$ peaks generated the $\alpha$-attactors and Horndeski general non-minimal derivative coupling (GNMDC) inflation introduced in Refs. [37] and [36] respectively. From the structural side the two inflationary models $[36,37]$ have a non-canonical kinetic term,

$$
\begin{aligned}
& \frac{\mathscr{L}^{\alpha-\text { attractors }}}{\sqrt{-g}}=\frac{R}{2}-\frac{\left(\partial_{\mu} \tilde{\phi}\right)^{2}}{2\left(1-\frac{\tilde{\phi}^{2}}{6 \alpha}\right)^{2}}+V(\tilde{\phi}) \\
& \frac{\mathscr{L}^{\mathrm{GNMDC}}}{\sqrt{-g}}=\frac{R}{2}-\hat{f}(\varphi) G^{\mu v} \partial_{\mu} \varphi \partial_{\nu} \varphi+V(\varphi)
\end{aligned}
$$

The special form of the $\alpha$-attractor potential and the GNMDC coupling can generate an enhanced 
$\mathscr{P}_{\mathscr{R}}(k)$ spectrum at some wavenumber $k_{\mathrm{p}}$ and the PBH formation becomes possible.

Fluctuations of the inflaton field $\phi$ in a homogeneous background induce scalar perturbations in the metric. In the comoving gauge we have $\delta \phi=0$ and $g_{i j}=a^{2}\left[(1-2 \mathscr{R}) \delta_{i j}+h_{i j}\right]$ and expanding the inflaton-gravity action to second order in $\mathscr{R}$ one obtains

$$
S_{(2)}=\frac{1}{2} \int \mathrm{d}^{4} x \sqrt{-g} a^{3} \frac{\dot{\phi}^{2}}{H^{2}}\left[\dot{\mathscr{R}}^{2}-\frac{\left(\partial_{i} \mathscr{R}\right)^{2}}{a^{2}}\right]
$$

After the variable redefinition $v=z \mathscr{R}$ where $z^{2}=a^{2} \dot{\phi}^{2} / H^{2}=2 a^{2} \varepsilon_{1}$ and switching to conformal time $\eta$ the Mukhanov-Sasaki equation for the evolution of the Fourier modes $v_{k}$ of $v(x)$ is found,

$$
v_{k}^{\prime \prime}+\left(k^{2}-\frac{z^{\prime \prime}}{z}\right) v_{k}=0
$$

where $z^{\prime \prime} / z$ is expressed in terms of the Hubble flow functions $\varepsilon_{1}, \varepsilon_{2}$ and $\varepsilon_{3}$ as

$$
\frac{z^{\prime \prime}}{z}=(a H)^{2}\left[2-\varepsilon_{1}+\frac{3}{2} \varepsilon_{2}-\frac{1}{2} \varepsilon_{1} \varepsilon_{2}+\frac{1}{4} \varepsilon_{2}^{2}+\frac{1}{2} \varepsilon_{2} \varepsilon_{3}\right]
$$

The power on a given scale of $\mathscr{R}$ is obtained once the solution $v_{k}$ of the Mukhanov-Sasaki equation is known and estimated at a time well after it exits the horizon and its value freezes out,

$$
\mathscr{P}_{\mathscr{R}}=\left.\frac{k^{3}}{2 \pi^{2}} \frac{\left|v_{k}\right|^{2}}{z^{2}}\right|_{k \ll a H}
$$

The initial conditions for the modes $v_{k}$ are set by the Bunch-Davies vacuum.
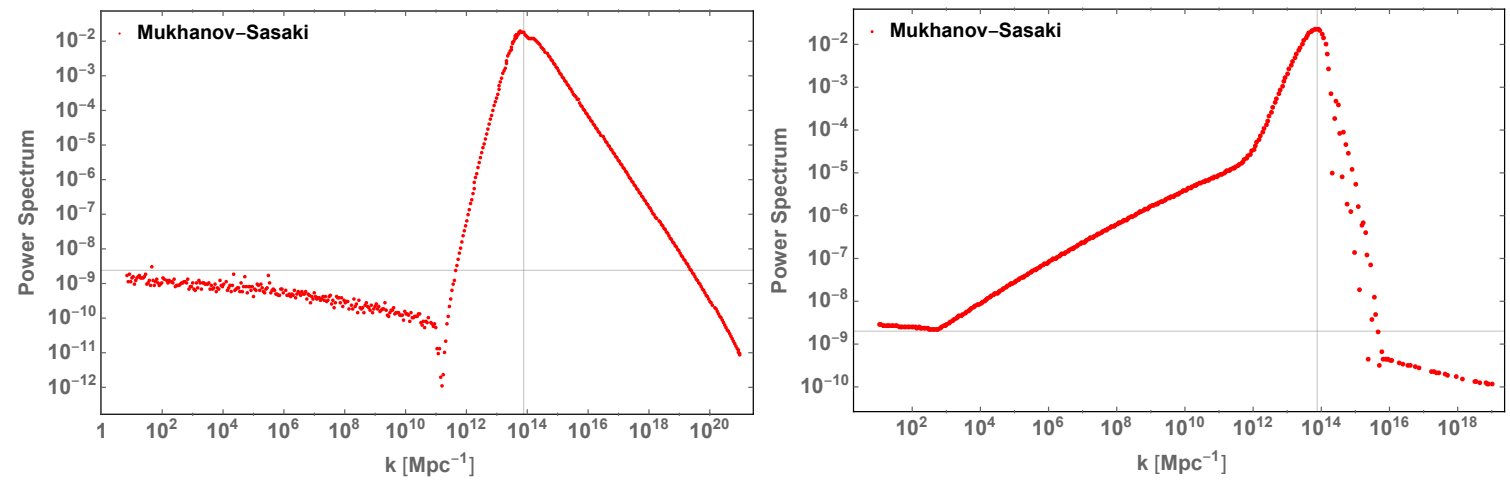

Figure 2: The plots depict the power spectra generated by the models (3.1) and (3.2) after solving numerically the Mukhanov-Sasaki equation for a thousand modes $k$.

The peak of the $\mathscr{P}_{\mathscr{R}}(k)$ satisfy the constraints given by Eq. (2.7), (2.8) and (2.9) for the reheating temperature predicted for each model, see Fig. 2. The position of the peak is motivated by the PBH dark matter scenario that we discuss in the next sections. Further details regarding the model building in the framework of $\alpha$-attractors can be found in Ref. [37,38] and for the generalnon minimal derivative coupling model in Ref. [36]. 


\section{PBHs as dark matter}

A PBH forms during a radiation dominated era if a preexisting overdensity with wavelength $k^{-1}$ and mass $M / \gamma$ enters the horizon after the reheating of the universe. The total abundance of PBHs with mass $M$ over the total dark matter (DM) abundance, $f_{\mathrm{PBH}}(M) \equiv \Omega_{\mathrm{PBH}}(M) / \Omega_{\mathrm{DM}}$, is expressed as

$$
f_{\mathrm{PBH}}(M)=\left(\frac{\beta(M)}{7.3 \times 10^{-15}}\right)\left(\frac{\Omega_{\mathrm{DM}} h^{2}}{0.12}\right)^{-1}\left(\frac{\gamma}{0.2}\right)^{\frac{3}{2}}\left(\frac{g\left(T_{k}\right)}{106.75}\right)^{-\frac{1}{4}}\left(\frac{M}{10^{20} \mathrm{~g}}\right)^{-1 / 2} .
$$

If the PBH form during the stage of oscillation of the inflaton field, i.e. matter domination era, the corresponding wavelength $k^{-1}$ enters the horizon before the complete decay of the inflaton and it is

$$
f_{\mathrm{PBH}}(M)=\left(\frac{\beta_{\mathrm{MD}}(M)}{2.1 \times 10^{-14}}\right)\left(\frac{\Omega_{\mathrm{DM}} h^{2}}{0.12}\right)^{-1}\left(\frac{\gamma_{\mathrm{M}}}{0.1}\right)^{\frac{3}{2}}\left(\frac{g\left(T_{\mathrm{rh}}\right)}{106.75}\right)^{-\frac{1}{4}}\left(\frac{M}{10^{20} \mathrm{~g}}\right)^{-1 / 2}\left(\frac{k}{k_{\mathrm{rh}}}\right)^{-3 / 2}
$$

The extra factor $\left(k / k_{\mathrm{rh}}\right)^{-3 / 2}$ accounts for the different redshift of the energy density of the the matter dominated universe compared to the radiation dominated universe.

In the mass window $M=10^{17}-5 \times 10^{22} \mathrm{~g}$ the $\mathrm{PBH}$ abundance can reach the maximum value, $f_{\mathrm{PBH}}=1$. Another motivated PBH mass range is $M=\mathscr{O}(1-100) M_{\odot}$ where LIGO detected several coalescence events the last years, although at that range it has to be $f_{\mathrm{PBH}} \lesssim 10^{-2}$. The models (3.1) and (3.2) can successfully generate PBHs with mass $M \sim 10^{18} \mathrm{~g}$ that can constitute a significant part of the dark matter in the galaxies. In the Fig. 3 we show the PBH abundance for the $\alpha$-attractors models for large and low reheating temperature.
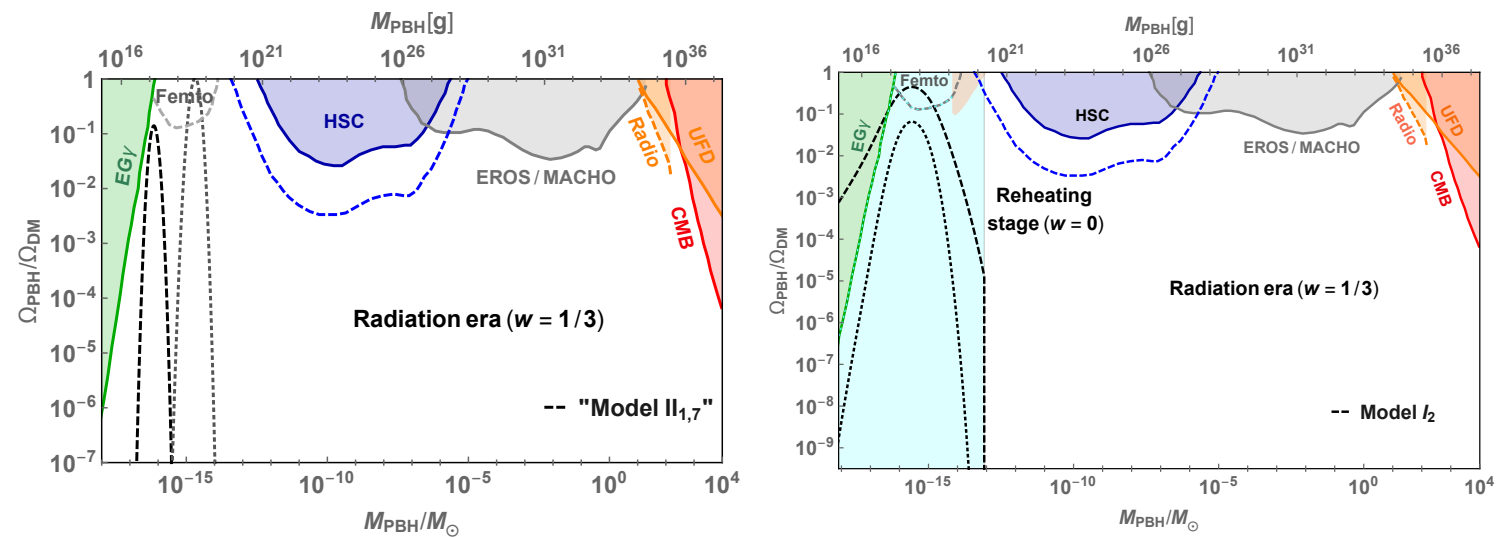

Figure 3: The fractional abundance of PBHs for $\alpha$-attractors models, produced during radiation (left panel) and during matter (right panel) for reheating temperature $T_{\mathrm{rh}} \simeq 10^{6} \mathrm{GeV}$. For details see Ref. [37]

\section{Remnants of PBHs as dark matter}

The formation of PBHs with mass $M$ can be realized only if the horizon mass right after inflation, $M_{\mathrm{end}}=4 \pi M_{\mathrm{Pl}}^{2} / H_{\mathrm{end}}$ is smaller than $M / \gamma$. The minimal PBH mass that can be generated 
is

$$
\frac{M}{\gamma} \gtrsim\left(\frac{r_{*}}{0.06}\right)^{-1 / 2} \text { grams }
$$

Corrections to the Hawking radiation emerging from some microscopic or quantum gravity theory that remove the infinity as $M \rightarrow 0$ imply that a stable or (meta)stable remnant might left behind after the evaporation. The mass of the final state of the evaporation can be written in terms of the Planck mass

$$
M_{\mathrm{rem}}=\kappa m_{\mathrm{Pl}} .
$$

The $\kappa$ is a factor that parameterizes our ignorance. Different theories predict stable black hole relics of different mass. The $\kappa$ may be of order one, with relic black hole masses characterized by the fundamental scale of gravity, $m_{\mathrm{Pl}}=G^{-2}$, but other values for the $\kappa$ are also admitted. In our analysis and expressions the $\kappa$ is a free parameter. This is a justified approach since we know nearly next to nothing about the physics at that energy scales.

The moment right after the evaporation the energy density of the PBHs has decreased $\left(\kappa m_{\mathrm{Pl}} / M\right)^{-1}$ times. This factor is much larger than one thus nearly the entire energy density of the initial PBHs turns into radiation apart from a tiny amount, reserved by the PBH remnants. The present density of the PBH remnants depends on the equation of state of the universe after the PBH evaporation. If we assume that a radiation domination phase follows the $\mathrm{PBH}$ evaporation the fractional abundance of the PBH remnants over the total DM abundance today is

$$
f_{\mathrm{rem}}(M)=\tilde{c} \beta\left(\frac{M_{\mathrm{eq}}}{M_{H}\left(t_{\mathrm{evap}}\right)}\right)^{1 / 2} \frac{\kappa m_{\mathrm{Pl}}}{M}\left(\frac{M}{m_{\mathrm{Pl}}}\right)^{\frac{4 w}{1+w}}
$$

where $\tilde{c}\left(\gamma, w, t_{\mathrm{eq}}\right)=2^{1 / 4} \tilde{h}\left(\gamma, w, t_{\mathrm{eq}}\right) \Omega_{\mathrm{m}} / \Omega_{\mathrm{DM}}$ and $M_{\mathrm{eq}}$ the horizon mass at the moment of radiationmatter equality. The assumption that there is a radiation domination phase after the PBH evaporation holds either when the universe has become $\mathrm{PBH}$ dominated before evaporation or when the equation of state of the background fluid is $w=1 / 3$.

The remnants abundance is maximal, $f_{\text {rem }}=1$, for $\kappa \gtrsim 10^{-18.5}$. PBHs remnants with smaller mass can constitute only a negligible amount of the total dark matter energy density. Hence, PBH remnants are possible to have a significant relic abundance only if they have mass

$$
M_{\mathrm{rem}} \gtrsim 10^{-24} \mathrm{~g},
$$

Note that $10^{-24} \mathrm{~g} \sim 1 \mathrm{GeV}$. Any object in the universe that comprises the dark matter in the galaxies and has mass less than $1 \mathrm{GeV}$ cannot be a black hole remnant. This is a conservative lower bound has been derived assuming the minimum possible $\mathrm{PBH}$ mass and the maximum possible formation rate, $\beta \sim 1$.

Let us discuss a particularly interesting cosmological scenario, that the bulk energy density is in the form of stiff fluid (SD era) with barotropic parameter $w=1$, called kination phase. Assuming that the SD era lasts at least until the moment of the evaporation and making the approximation of instantaneous evaporation, the energy density of the PBHs at that moment, $t_{\mathrm{evap}}^{<}$, is

$$
\frac{\rho_{\mathrm{PBH}}\left(t_{\text {evap }}^{<}\right)}{\rho_{\mathrm{S}}\left(t_{\text {evap }}^{<}\right)}=\frac{3}{2} \gamma^{2} \beta \frac{M^{2}}{m_{\mathrm{Pl}}^{2}} .
$$


The assumption of a kination phase is valid roughly for $\gamma^{2} \beta M^{2} m_{\mathrm{Pl}}^{-2}<1$, otherwise the universe becomes PBH dominated before the moment of evaporation. Assuming that the evaporation products thermalize fast, the radiation redshifts like $\rho_{\text {rad }} \propto g_{*} g_{s}^{-4 / 3} a^{-4}$ whereas the stiff fluid background redshifts like $\rho_{\mathrm{S}} \propto a^{-6}$. At some moment the radiation dominates the background energy density and we define it as the reheating moment $t_{\mathrm{rh}}$. We also define the reheating temperature of the universe that reads

$$
T_{\text {rh }} \equiv 6.3 \mathrm{MeV}\left(\frac{\beta}{10^{-28}}\right)^{3 / 4} \gamma^{3 / 2} g_{*}^{-1 / 2}
$$

Until the moment $t_{\mathrm{rh}}$ the energy density of the PBH remnants increases relatively to the stiff fluid dominated background as $\rho_{\mathrm{rem}} / \rho_{\mathrm{S}} \propto a^{3}$ and afterwards, that radiation dominates, it increases as $\rho_{\text {rem }} / \rho_{\text {rad }} \propto T^{-1}$. We find that the fractional abundance of the PBH remnants is

$$
f_{\mathrm{rem}}(M) \simeq 4 \kappa \sqrt{\gamma}\left(\frac{\beta}{10^{-32}}\right)^{1 / 4}\left(\frac{M}{10^{5} \mathrm{~g}}\right)^{-2} .
$$

For $\kappa \sim 1$ and $M \sim 10^{5} \mathrm{~g}, \beta$ values as small as $10^{-32}$ can explain the observed dark matter in the universe. Pressure is maximal and we expect the overdense regions to be spherically symmetric when the density perturbation becomes larger than $\delta_{c}=0.375$. For the formation probability $\beta(M)$ given by the Eq. (2.2) we find that power spectrum values $\mathscr{P}_{\mathscr{R}} \lesssim 3.5 \times 10^{-3}$, for $\kappa \gtrsim 1$ and $M \sim 10^{5} \mathrm{~g}$, can yield $f_{\text {rem }}=1$.

The construction of kination inflation models that induce the PBH production is very challenging. Firstly, the inflaton runs away until it freezes at some value $\varphi_{F}$ and this residual potential energy of the inflaton must not to spoil the early and late time cosmology. The inflaton potential energy at $\varphi_{F}$ has to be tuned to values $V\left(\varphi_{F}\right) \lesssim 10^{-120} M_{\mathrm{Pl}}^{4}$, similarly to all the quintessence models. Secondly, the kination inflaton model parameters are self-constrained. A particular PBH mass $M$ specifies the $k$ of the $\mathscr{P}_{\mathscr{R}}(k)$ only if the reheating temperature is known. However, the reheating temperature is not a free parameter, as e.g in matter or radiation cases where the $T_{\mathrm{rh}}$ depends on the inflaton decay rate. In the kination scenario the $T_{\mathrm{rh}}$ depends on the $\beta$. The $\beta$ is found by the condition $f_{\text {rem }}=1$ and this fixes the reheating temperature.

Hence, the characteristics of the peak in the power spectrum determine

i) the mass of the evaporating PBHs,

ii) the dark matter abundance, and

iii) the reheating temperature of the universe.

In addition, the tail of the potential might lead to the observed late time acceleration of the universe. Undoubtedly, this scenario is remarkably economic, see Fig. 4.

\section{Constraints on the primordial curvature perturbations No2: Induced GWs}

The primordial density scalar perturbations unambiguously source tensor modes at second order perturbation theory [39-43], the so-called induced gravitational waves (IGW). The cosmological implications of the scalar induced GWs to second order were firstly discussed in the pioneering 

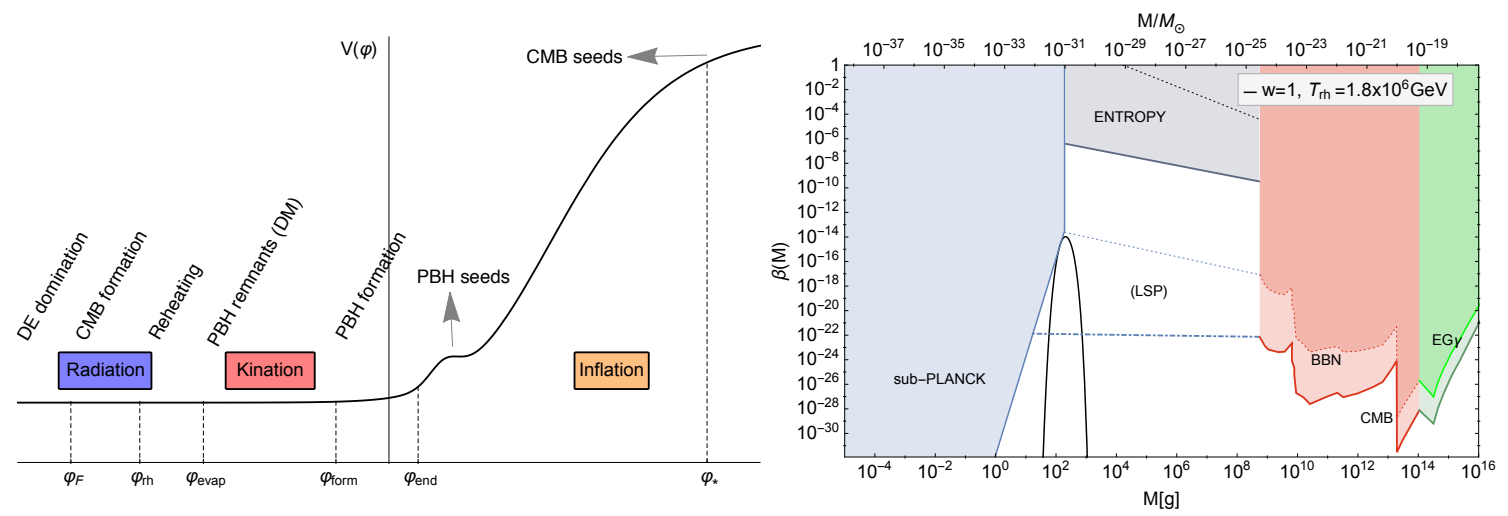

Figure 4: Left panel. A schematic illustration of the runaway inflationary model introduced (kination scenario) that produces PBHs, explains the dark matter with the PBH remnants, reheats the Universe via the PBH evaporation and implements a wCDM late time cosmology. Right panel. The $\beta(M)$ predicted by the kination inflationary model. The PBHs with central mass $M=2 \times$ $10^{2} \mathrm{~g}$ are produced and evaporate during the kination era (SD). The evaporation leaves behind subplanckian mass remnants with mass $M_{\mathrm{rem}} \simeq 6 \times 10^{8} \mathrm{GeV}$ that comprise the entire dark matter, $f_{\text {rem }}=1$.

works [44], where the effetcs of IGWs on CMB polarization was computed, and [45], where the spectrum of the IGWs on small scales was studied. Further notable results followed that extended our understanding and formulation of IGW physics [46-48] and gave us insights of how to probe the primordial power spectrum, $\mathscr{P}_{\mathscr{R}}(k)$, at small scales.

The new observational window to the very early universe through the IGWs can test scenarios that exhibit an enhanced power at small scales and predict PBHs. The interpretation of a GW signal is nevertheless a non-trivial task and one needs to take into account several parameters, such as the reheating temperature and the equation of state of the early universe. From a different point of view, detection of the relic GWs stochastic background is a direct probe of the very early cosmic history, which is unknown for $t \lesssim 1 \mathrm{~s}$ [30].

Considering the field equations of general relativity for a single perfect cosmological fluid with the standard density-pressure equation of state relation $p=w \rho$, the evolution equations for the gravitational potential is obtained

$$
\Phi^{\prime \prime}(x)+3(1+w) \mathscr{H}(\eta) \Phi^{\prime}(x)+w k^{2} \Phi(x)=0 .
$$

The evolution of the induced tensor modes is given by the equation,

$$
h_{\mathbf{k}}{ }^{\prime \prime}(\eta)+2 \mathscr{H}(\eta) h_{\mathbf{k}}{ }^{\prime}(\eta)+k^{2} h_{\mathbf{k}}(\eta)=\mathscr{S}_{\mathbf{k}}(\eta),
$$

where the source function $\mathscr{S}_{\mathbf{k}}$ at the left hand side plays a critical role. It is a convolution of scalar perturbations at different wavenumbers given by,

$$
\begin{array}{r}
\mathscr{S}_{\mathbf{k}}(\eta)=4 \int \frac{d^{3} q}{(2 \pi)^{3 / 2}} e^{i j}(\mathbf{k}) q_{i} q_{j} \phi_{\mathbf{k}} \phi_{\mathbf{k}-\mathbf{q}} \\
\times f(|\mathbf{q}| / k,|\mathbf{k}-\mathbf{q}| / k, \eta, k) .
\end{array}
$$


Here, $e^{i j}$ is the polarization tensor, and $f$ is an auxiliary function. A useful definition for the energy density of IGWs per unit logarithmic frequency interval is given in terms of the tensor power spectrum as,

$$
\Omega_{\mathrm{IGW}}(\eta, k) \equiv \frac{1}{24}\left(\frac{k}{a H}\right)^{2} \overline{\mathscr{P}_{h}(\eta, k)},
$$

where the over-line denotes the oscillation average. The power spectral density of the tensor perturbations is expressed as a double integral involving the power spectrum of the curvature perturbations,

$$
\overline{\mathscr{P}_{h}(\eta, k)}=\int_{0}^{\infty} d v \int_{|1-v|}^{1+v} d u \mathscr{T}(u, v, \eta, k) \mathscr{P}_{\mathscr{R}}(u k) \mathscr{P}_{\mathscr{R}}(v k)
$$

where $\mathscr{T}$ is the tensor transfer function. The formula for the density parameter of IGWs produced in the RD era can be found in the literature, see e.g. Ref. [49] . For the early kination era with transition to radiation, $\mathrm{eKD} \rightarrow \mathrm{RD}$, the density parameter of IGWs is given by [50]

$$
\begin{gathered}
\Omega_{\mathrm{IGW}}^{\mathrm{eKD} \rightarrow \mathrm{RD}}\left(\eta_{\mathrm{c}}, k\right)=\frac{1}{6} A_{\mathscr{R}}^{2}\left(\frac{k_{\mathrm{p}}}{a H\left(\eta_{\mathrm{c}}\right)}\right)^{2}\left[1-\left(\frac{k}{2 k_{\mathrm{p}}}\right)^{2}\right]^{2} \\
\times \overline{I_{\mathrm{eKD} \rightarrow \mathrm{RD}}^{2}\left(\frac{k_{\mathrm{p}}}{k}, \frac{k_{\mathrm{p}}}{k}, \eta_{\mathrm{c}}, k\right)} \Theta\left(1-\frac{k}{2 k_{\mathrm{p}}}\right) .
\end{gathered}
$$

The $\mathscr{A}_{\mathscr{R}}$ is the amplitude of the curvature power spectrum at $k=k_{\mathrm{p}}$ and $\eta_{c}$ is the moment the amplitude of the IGWs freezes out. The unit step function $\Theta$ is included by conservation of momentum, so that tensor modes with $k>2 k_{\mathrm{p}}$ are cut-off.

The spectral shape for the $\Omega_{\mathrm{IGW}}\left(t_{0}, f\right)$, depends on the features of the source, the scalar spectrum $\mathscr{P}_{\mathscr{R}}(k)$. It maximizes at a frequency $f_{\mathrm{IGW}}^{\mathrm{p}}$, in a little offset from $k_{\mathrm{p}} / 2 \pi$, depending on the width of the $\mathscr{P}_{\mathscr{R}}(k)$ [50,51]. It is interesting to mention that, for the radiation domination case at least, the $\mathscr{P}_{\mathscr{R}}(k)$ shape is projected in a much more informative manner onto the IGW spectrum than on the PBH mass distribution, which is predominantly monochromatic. By observing the IGW spectral shape and the power law scaling in the large- $f$ band one can infer the width and the amplitude of the scalar spectrum, the generator of the IGWs, see Fig. 5.

Consequently, we can say that the detection of the IGW spectrum is a portal to the primordial power spectrum of curvature perturbations, $\mathscr{P}_{\mathscr{R}}(k)$. For further analysis and comments we refer the reader to Ref. [50] where explicit scalar spectra and inflationary models are discussed and discriminated.

\section{Conclusions}

The gravitational observation of black hole mergers by LIGO [52] offers us an unprecedented piece of information about the dark sector of the universe. It has motivated cosmologists to investigate the scenario that PBHs comprise a significant fraction of the dark matter in the universe and, additionally, search for the amplitude of the primordial density perturbations at small scales far beyond the scales directly accessible in the CMB [53].

Here, I presented the implications of the evaporating PBHs on the variance of the density perturbations for different reheating temperatures and stressed that the scalar power spectrum gen- 

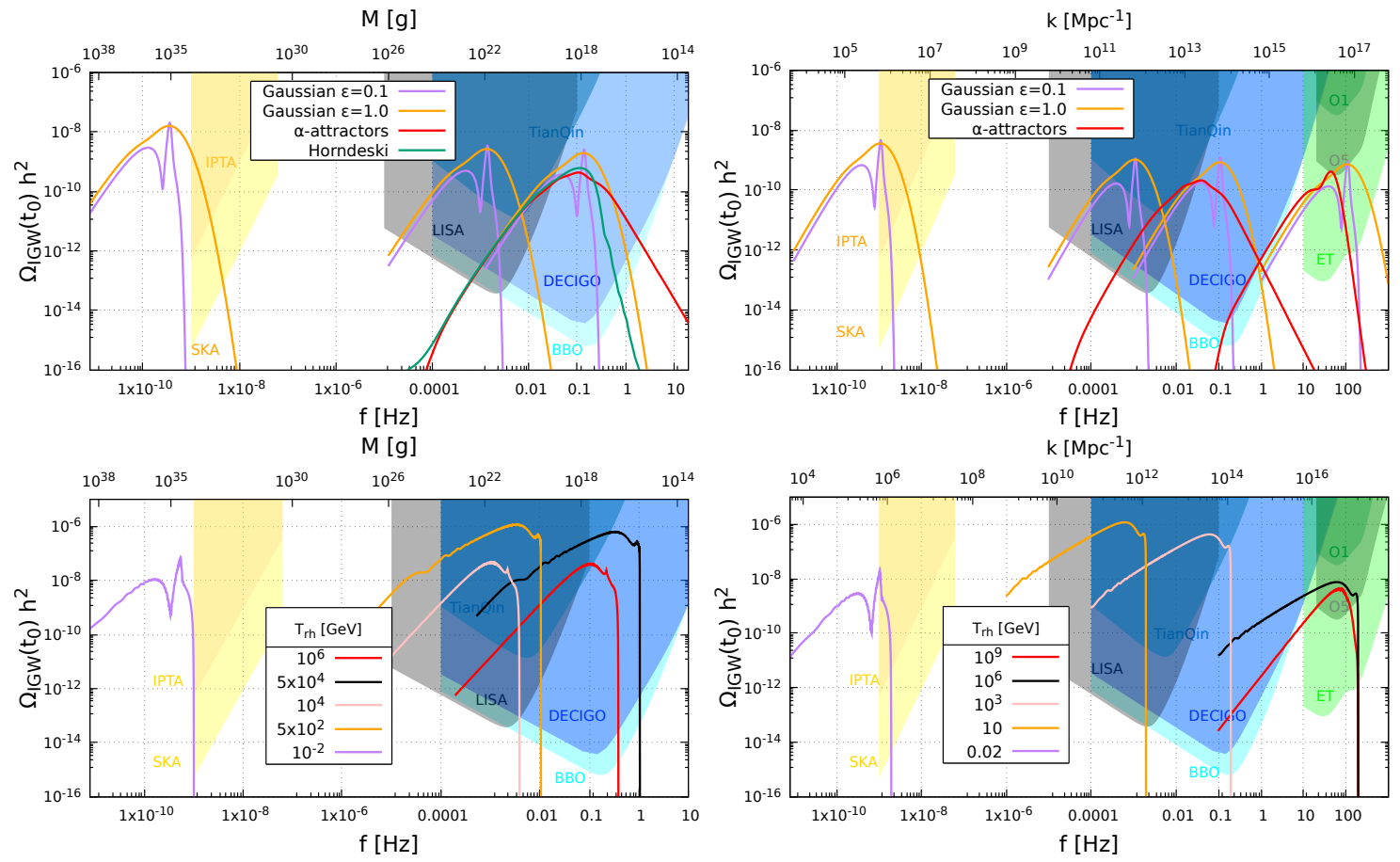

Figure 5: The IGW spectral shapes for two early universe scenarios, the RD (top panels) and the $\mathrm{eKD} \rightarrow \mathrm{RD}$ (bottom panels) for different reheating temperatures. In the left panels we consider the scenario of abundant PBH production and in the right panels negligible PBH abundances. In the background of the IGW spectral curves the sensitivity curves of current/planned/proposed GW detectors are shown.

erated by any inflationary model has to satisfy a specific constraint in the large $k$ band of the spectrum.

In the following I introduced the primordial black hole production scenario from inflationary models. I presented inflationary models built in the framework of $\alpha$-attractors and inflation models with general non-minimal derivative coupling. I also discussed the cosmology of mini primordial black holes, motivated by the theoretical postulation that a stable or long lived remnant is left behind after the evaporation of the "black" holes. Mini PBHs imply that the comoving curvature perturbation is enhanced at the extreme end of the $\mathscr{P}_{\mathscr{R}}(k)$. This is a rather attractive feature since the required large primordial inhomogeneities can be produced by the inflationary phase without spoiling the spectral index value $n_{s}$. A particularly interesting and novel scenario is that where the $\mathrm{PBH}$ production takes place during a kination regime. A kination regime takes place if the inflaton potential has no minimum and the inflaton runs away after the end of inflation. The radiation is produced by the $\mathrm{PBH}$ evaporation that gradually dominates the energy density and reheats the universe. The PBHs remnants can account for the entire dark matter of the universe. Interestingly enough, the non-decaying inflaton can additionally act as quintessence field giving rise to the observed late time accelerated expansion implementing a wCDM cosmological model.

Finally, I discussed the shape of the induced GWs produced in the early universe assuming different scenarios and models. I focused on a $\mathscr{P}_{\mathscr{R}}(k)$ shape with a peak, took into account the 
transition details and I targeted at cosmological parameter values that can be tested by the existing and designed GW experiments, such as LIGO and LISA. The detection of the induced GW spectrum can be used to discriminate inflationary models, hence it is a portal to the primordial power spectrum of curvature perturbations, $\mathscr{P}_{\mathscr{R}}(k)$.

\section{Acknowledgments}

This work is supported by IKY Scholarship, co-financed by Greece and the European Union (European Social Fund-ESF), through the Operational Program "Human Resources Development, Education and Lifelong Learning" in the context of the project "Reinforcement of Postdoctoral Researchers" - 2nd Cycle (MIS-5033021), implemented by the State Scholarships Foundation.

\section{References}

[1] B. J. Carr, “The Primordial black hole mass spectrum,” Astrophys. J. 201 (1975), 1-19

[2] S. W. Hawking, "Particle Creation by Black Holes," Commun. Math. Phys. 43 (1975), 199-220

[3] S. W. Hawking, "Black hole explosions," Nature 248 (1974), 30-31

[4] B. V. Vainer and P. D. Naselskii, Sov. Astron. 22, (1978) 138 [Astron. Zh. 55 (1978) 231]

[5] Y. B. ZelâĂŹdovich, A. A. Starobinskii, M. I. Khlopov, and V. M. Chechetkin, Sov. Astron. Lett., 3, (1977) 110 [Pisma Astron. Zh. 3 (1977) 208]

[6] S. Miyama and K. Sato, Prog. Theor. Phys. 59 (1978) 1012. doi:10.1143/PTP.59.1012

[7] B. V. Vainer, O. V. Dryzhakova, and P. D. Naselskii, Sov.Astron.Lett.,4, (1978) 185 [Pisma Astron. Zh. 4 (1978) 344]

[8] D. Lindley, Mon. Not. R. Astron. Soc. 193, 593 (1980).

[9] K. Kohri and J. Yokoyama, "Primordial black holes and primordial nucleosynthesis. 1. Effects of hadron injection from low mass holes,” Phys. Rev. D 61 (2000), 023501 [arXiv:astro-ph/9908160 [astro-ph]].

[10] M. Kawasaki, K. Kohri and T. Moroi, "Big-Bang nucleosynthesis and hadronic decay of long-lived massive particles,” Phys. Rev. D 71 (2005), 083502 [arXiv:astro-ph/0408426 [astro-ph]].

[11] B. J. Carr, K. Kohri, Y. Sendouda and J. Yokoyama, "New cosmological constraints on primordial black holes,” Phys. Rev. D 81 (2010), 104019 [arXiv:0912.5297 [astro-ph.CO]].

[12] L. Zhang, X. Chen, M. Kamionkowski, Z. g. Si and Z. Zheng, Phys. Rev. D 76 (2007), 061301 doi:10.1103/PhysRevD.76.061301 [arXiv:0704.2444 [astro-ph]].

[13] D. N. Page and S. W. Hawking, “Gamma rays from primordial black holes,” Astrophys. J. 206 (1976), $1-7$

[14] J. H. MacGibbon and B. J. Carr, “Cosmic rays from primordial black holes,” Astrophys. J. 371 (1991), 447-469

[15] B. J. Carr and J. H. MacGibbon, "Cosmic rays from primordial black holes and constraints on the early universe,” Phys. Rept. 307 (1998), 141-154

[16] A. Barrau, G. Boudoul and L. Derome, "An improved gamma-ray limit on the density of pbhs," [arXiv:astro-ph/0304528 [astro-ph]]. 
[17] B. J. Carr, K. Kohri, Y. Sendouda and J. Yokoyama, "Constraints on primordial black holes from the Galactic gamma-ray background,” Phys. Rev. D 94 (2016) no.4, 044029 [arXiv:1604.05349 [astro-ph.CO]].

[18] A. Barnacka, J. F. Glicenstein and R. Moderski, "New constraints on primordial black holes abundance from femtolensing of gamma-ray bursts," Phys. Rev. D 86 (2012), 043001 [arXiv:1204.2056 [astro-ph.CO]].

[19] P. Tisserand et al. [EROS-2], "Limits on the Macho Content of the Galactic Halo from the EROS-2 Survey of the Magellanic Clouds," Astron. Astrophys. 469 (2007), 387-404 [arXiv:astro-ph/0607207 [astro-ph]].

[20] H. Niikura, M. Takada, N. Yasuda, R. H. Lupton, T. Sumi, S. More, T. Kurita, S. Sugiyama, A. More, M. Oguri and M. Chiba, "Microlensing constraints on primordial black holes with Subaru/HSC Andromeda observations," Nature Astron. 3 (2019) no.6, 524-534 [arXiv:1701.02151 [astro-ph.CO]].

[21] M. Ricotti, J. P. Ostriker and K. J. Mack, "Effect of Primordial Black Holes on the Cosmic Microwave Background and Cosmological Parameter Estimates,” Astrophys. J. 680 (2008), 829 [arXiv:0709.0524 [astro-ph]].

[22] B. Carr, F. Kuhnel and M. Sandstad, "Primordial Black Holes as Dark Matter," Phys. Rev. D 94 (2016) no.8, 083504 [arXiv:1607.06077 [astro-ph.CO]].

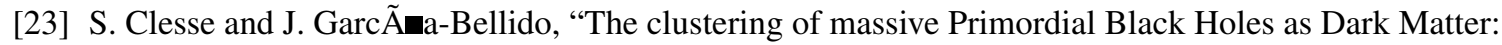
measuring their mass distribution with Advanced LIGO,” Phys. Dark Univ. 15 (2017), 142-147 [arXiv:1603.05234 [astro-ph.CO]].

[24] S. Bird, I. Cholis, J. B. MuÃśoz, Y. Ali-HaÃŕmoud, M. Kamionkowski, E. D. Kovetz, A. Raccanelli and A. G. Riess, “Did LIGO detect dark matter?," Phys. Rev. Lett. 116 (2016) no.20, 201301 [arXiv:1603.00464 [astro-ph.CO]].

[25] D. Gaggero, G. Bertone, F. Calore, R. M. T. Connors, M. Lovell, S. Markoff and E. Storm, "Searching for Primordial Black Holes in the radio and X-ray sky,” Phys. Rev. Lett. 118 (2017) no.24, 241101 [arXiv:1612.00457 [astro-ph.HE]].

[26] B. Carr, K. Kohri, Y. Sendouda and J. Yokoyama, “Constraints on Primordial Black Holes," [arXiv:2002.12778 [astro-ph.CO]].

[27] T. Harada, C. M. Yoo and K. Kohri, “Threshold of primordial black hole formation,” Phys. Rev. D 88 (2013) no.8, 084051 [arXiv:1309.4201 [astro-ph.CO]].

[28] T. Harada, C. M. Yoo, K. Kohri, K. i. Nakao and S. Jhingan, "Primordial black hole formation in the matter-dominated phase of the Universe,” Astrophys. J. 833 (2016) no.1, 61 [arXiv:1609.01588 [astro-ph.CO]].

[29] T. Harada, C. M. Yoo, K. Kohri and K. I. Nakao, "Spins of primordial black holes formed in the matter-dominated phase of the Universe," Phys. Rev. D 96 (2017) no.8, 083517 [arXiv:1707.03595 [gr-qc]].

[30] R. Allahverdi, M. A. Amin, A. Berlin, N. Bernal, C. T. Byrnes, M. Sten Delos, A. L. Erickcek, M. Escudero, D. G. Figueroa, K. Freese, T. Harada, D. Hooper, D. I. Kaiser, T. Karwal, K. Kohri, G. Krnjaic, M. Lewicki, K. D. Lozanov, V. Poulin, K. Sinha, T. L. Smith, T. Takahashi, T. Tenkanen, J. Unwin, V. Vaskonen and S. Watson, "The First Three Seconds: a Review of Possible Expansion Histories of the Early Universe," [arXiv:2006.16182 [astro-ph.CO]].

[31] I. Dalianis, "Constraints on the curvature power spectrum from primordial black hole evaporation," JCAP 08 (2019), 032 [arXiv:1812.09807 [astro-ph.CO]]. 
[32] J. Garcia-Bellido and E. Ruiz Morales, "Primordial black holes from single field models of inflation," Phys. Dark Univ. 18 (2017), 47-54 [arXiv:1702.03901 [astro-ph.CO]].

[33] G. Ballesteros and M. Taoso, Phys. Rev. D 97 (2018) no.2, 023501 doi:10.1103/PhysRevD.97.023501 [arXiv:1709.05565 [hep-ph]].

[34] C. Germani and A. Kehagias, "New Model of Inflation with Non-minimal Derivative Coupling of Standard Model Higgs Boson to Gravity,” Phys. Rev. Lett. 105 (2010), 011302 [arXiv:1003.2635 [hep-ph]].

[35] C. Fu, P. Wu and H. Yu, "Primordial Black Holes from Inflation with Nonminimal Derivative Coupling,” Phys. Rev. D 100 (2019) no.6, 063532 [arXiv:1907.05042 [astro-ph.CO]].

[36] I. Dalianis, S. Karydas and E. Papantonopoulos, "Generalized Non-Minimal Derivative Coupling: Application to Inflation and Primordial Black Hole Production,” JCAP 06 (2020), 040 [arXiv:1910.00622 [astro-ph.CO]].

[37] I. Dalianis, A. Kehagias and G. Tringas, "Primordial black holes from $\alpha$-attractors," JCAP 01 (2019), 037 [arXiv:1805.09483 [astro-ph.CO]].

[38] I. Dalianis and G. Tringas, "Primordial black hole remnants as dark matter produced in thermal, matter, and runaway-quintessence postinflationary scenarios,” Phys. Rev. D 100 (2019) no.8, 083512 [arXiv:1905.01741 [astro-ph.CO]].

[39] S. Matarrese, O. Pantano and D. Saez, "A General relativistic approach to the nonlinear evolution of collisionless matter,” Phys. Rev. D 47 (1993), 1311-1323

[40] S. Matarrese, S. Mollerach and M. Bruni, "Second order perturbations of the Einstein-de Sitter universe,” Phys. Rev. D 58 (1998), 043504 [arXiv:astro-ph/9707278 [astro-ph]].

[41] H. Noh and J. c. Hwang, "Second-order perturbations of the Friedmann world model," Phys. Rev. D 69 (2004), 104011

[42] C. Carbone and S. Matarrese, "A Unified treatment of cosmological perturbations from super-horizon to small scales,” Phys. Rev. D 71 (2005), 043508 [arXiv:astro-ph/0407611 [astro-ph]].

[43] K. Nakamura, "Second-order gauge invariant cosmological perturbation theory: Einstein equations in terms of gauge invariant variables," Prog. Theor. Phys. 117 (2007), 17-74 [arXiv:gr-qc/0605108 [gr-qc]].

[44] S. Mollerach, D. Harari and S. Matarrese, "CMB polarization from secondary vector and tensor modes,” Phys. Rev. D 69 (2004), 063002 [arXiv:astro-ph/0310711 [astro-ph]].

[45] K. N. Ananda, C. Clarkson and D. Wands, "The Cosmological gravitational wave background from primordial density perturbations,” Phys. Rev. D 75 (2007), 123518 [arXiv:gr-qc/0612013 [gr-qc]].

[46] H. Assadullahi and D. Wands, "Gravitational waves from an early matter era," Phys. Rev. D 79 (2009), 083511 [arXiv:0901.0989 [astro-ph.CO]].

[47] D. Baumann, P. J. Steinhardt, K. Takahashi and K. Ichiki, "Gravitational Wave Spectrum Induced by Primordial Scalar Perturbations,” Phys. Rev. D 76 (2007), 084019 [arXiv:hep-th/0703290 [hep-th]].

[48] R. Saito and J. Yokoyama, "Gravitational wave background as a probe of the primordial black hole abundance,” Phys. Rev. Lett. 102 (2009), 161101 [arXiv:0812.4339 [astro-ph]].

[49] K. Kohri and T. Terada, "Semianalytic calculation of gravitational wave spectrum nonlinearly induced from primordial curvature perturbations,” Phys. Rev. D 97 (2018) no.12, 123532 [arXiv:1804.08577 [gr-qc]]. 
[50] I. Dalianis and K. Kritos, "Exploring the Spectral Shape of Gravitational Waves Induced by Primordial Scalar Perturbations and Connection with the Primordial Black Hole Scenarios," [arXiv:2007.07915 [astro-ph.CO]].

[51] R. G. Cai, S. Pi and M. Sasaki, "Universal infrared scaling of gravitational wave background spectra," [arXiv:1909.13728 [astro-ph.CO]].

[52] B. P. Abbott et al. [LIGO Scientific and Virgo Collaborations], "Binary Black Hole Mergers in the first Advanced LIGO Observing Run,” Phys. Rev. X 6, no. 4, 041015 (2016) Erratum: [Phys. Rev. X 8, no. 3, 039903 (2018)] [arXiv:1606.04856 [gr-qc]].

[53] M. Sasaki, T. Suyama, T. Tanaka and S. Yokoyama, "Primordial black hole- perspectives in gravitational wave astronomy,” Class. Quant. Grav. 35 (2018) no.6, 063001 [arXiv:1801.05235 [astro-ph.CO]]. 